\title{
The Relationship Between Serum Levels of Interleukins 6, 8,10 and Clinical Outcome in Patients With Severe Traumatic Brain Injury
}

\author{
Sharhokh Yousefzadeh-Chabok ${ }^{1}$; Anoush Dehnadi Moghaddam ${ }^{2}$; Ehsan Kazemnejad- \\ Leili ${ }^{3}$; Zahra Saneei ${ }^{4}$; Marieh Hosseinpour ${ }^{4}$; Leila Kouchakinejad-Eramsadati ${ }^{5}$; Alireza \\ Razzaghi ${ }^{6}$; Zahra Mohtasham-Amiri ${ }^{4, *}$ \\ ${ }_{1}^{1}$ Neurosurgery Department, Guilan Road Trauma Research Center, Guilan University of Medical Sciences, Rasht, IR Iran \\ ${ }_{3}^{2}$ Anesthesiology Department, Guilan Road Trauma Research Center, Guilan University of Medical Sciences, Rasht, IR Iran \\ 3 Biostatistics Department,Guilan Road Trauma Research Center, Guilan University of Medical Sciences, Rasht, IR Iran \\ ${ }_{5}^{4}$ Guilan Road Trauma Research Center, Guilan University of Medical Sciences, Rasht, IR Iran \\ ${ }_{5}^{5}$ Human Resource Management, Guilan Road Trauma Research Center, Guilan University of Medical Sciences, Rasht, IR Iran \\ 6 Guilan Road Trauma Research Center, Guilan University of Medical Sciences, Rasht, IR Iran \\ *Corresponding author:Zahra Mohtasham-Amiri, Guilan Road Trauma Research Center, Guilan University of Medical Sciences, Rasht, IR Iran. Tel: +98-1316690006, Fax:+98-1317753836, \\ E-mail:mohtasham@gums.ac.ir
}

Received: March 1, 2014; Revised: January 4, 2015; Accepted: February 7, 2015

\begin{abstract}
Background: Clinical outcome in patients with severe traumatic brain injury (TBI) depends on both primary and secondary brain injuries. Neuroinflammation is an important secondary mechanism, which occurs by releasing interleukins (ILs). Increased levels of ILs may affect clinical outcome following TBI.

Objectives: This study aimed to determine the relationship between the serum levels of interleukins 6, 8 and 10 and clinical outcome in patients with severe TBI 6 months after injury.

Patients and Methods:In a descriptive-analytical study, 44 patients with GCS $\leq 8$ (Glasgow coma scale) and age $\geq 14$ years were included. Their blood samples were collected at first 6 hours after injury. Clinical outcome was determined based on GOS (Glasgow Outcome Scale) at 6 months after head injury. Serum levels of interleukins 6, 8 and 10 were measured using the ELISA method. Spearman's rho, independent T-Test, and Mann-Whitney Test were used for data analysis.

Results: Comparing the serum levels of interleukins in two groups with favorable and unfavorable clinical outcomes showed that the mean serum levels of interleukins 6 and 8 in group with favorable outcome was $85.2 \pm 51.6$ and $52.2 \pm 31.9$, respectively lower than those of group with unfavorable outcome with $162.3 \pm 141.1$ and $173.6 \pm 257.3(\mathrm{P}<0.03)$ and $(\mathrm{P}<0.01)$.

Conclusions: Increased serum levels of interleukins 6 and 8 as a predictive marker might be associated with unfavorable clinical outcome in patients with severe TBI.
\end{abstract}

Keywords: Head Injury; Interleukin; Brain

\section{Background}

Traumatic brain injury (TBI) is one of the leading causes of morbidity and mortality worldwide particularly among young people and imposes high costs directly and indirectly on societies (1). Based on reports provided by United States Centers for Disease Control and Prevention, 1.7 million victims suffer from traumatic brain injury and its complications (2). Studies have shown that the outcome of patients with TBI is determined by severity of primary injury and extent of secondary brain damage (SBD) (3). The mechanisms of primary injury are caused by mechanical injury to neurons, axons, glia and blood vessels. These effects induce secondary mechanisms which result from depolarization (4), neurotransmitters release (5), lipids disturbance (6) and delayed inflammatory and immunological response in minutes, days and even months after the primary injury (7). Inflammation following TBI can cause complications which may affect patient outcome including systemic inflammatory response syndrome (SIRS). This clinical syndrome is the result of extended activity of inflammatory pathways (8). Activated inflammatory cascade after TBI is mediated by release of pre and anti-inflammatory cytokines. These cytokines are polypeptides that are rarely observed in a healthy brain but their level rises rapidly in response to pathological or stressful conditions $(8,9)$. There is different types of cytokines including interleukin. Studies have indicated the pathological role of interleukins in both peripheral and central nervous system (CNS) diseases such as shock, meningitis and head injury (10). Developments on understanding secondary injury provide new therapeutic intervention to prevent progressive tissue damage and following dysfunction. To obtain effective neuro

Copyright (C) 2015, Kashan University of Medical Sciences. This is an open-access article distributed under the terms of the Creative Commons Attribution-NonCommercial 4.0 International License (http://creativecommons.org/licenses/by-nc/4.0/) which permits copy and redistribute the material just in noncommercial usages, provided the original work is properly cited. 
protective treatments in TBI patients, understanding of complex cellular and molecular events which contribute to secondary injury is important. Neuro inflammation is a major secondary injury mechanism after TBI which results in post-trauma sustained damage. However, experimental and clinical research proved that neuro inflammation after TBI can have both detrimental and beneficial effects which are probably different in acute and delayed phases. The key to developing future anti-inflammatory based neuro protective treatments for TBI is to reduce the detrimental and neurotoxic effects of neuro inflammation while promoting the beneficial and neurotropic effects, thereby creating optimal conditions for regeneration and repair after injury $(11,12)$. Many studies revealed that released interleukins affect clinical outcomes after brain injury and cause pre-inflammatory changes $(13,14)$. Interleukin 8 released from monocytes and endothelial cells involve in degeneration and regeneration $(14,15)$. Interleukin 10 produced by macrophage and microglia has anti-inflammatory and immunosuppressant effect (14). Despite of significant improvements in organizing measures related to traumatic brain injury to date, no effective treatment is available for these patients so far and the rate of mortality and morbidity is high (1). Although acute inflammation plays an important role in pathophysiology of brain, the special relationship between mediators of inflammation and patient outcome after TBI has not been fully established yet (16).

\section{Objectives}

The aim of this study was to achieve a better understanding of the effects of a number of interleukins which are inflammatory mediators on clinical outcome by analyzing their serum levels in patients with severe TBI.

\section{Patients and Methods}

This is a descriptive-analytical study conducted on 47 TBI patients with GCS $\leq 8$, admitted to Poursina Teaching Hospital in Rasht (Guilan province). Out of total admitted patients, 3 were excluded because they were younger than 14 years old. The patients with underlying diseases were excluded from the study. The patients showed no additional medical problems during the time between the sampling and assessing the outcome. Blood samples were taken at first six hours after injury from 44 remaining patients, then the samples were centrifuged and the supernatant was immediately transferred to $-80^{\circ} \mathrm{C}$ freezer and kept there until the test time. Measuring concentrations of interleukin 6, 8 and 10 in blood serum of patients was performed by ELISA (Enzyme Liked Immunosorbent Assay), which is a high sensitive method (Bender Med System Co). Patients outcomes were determined 6 months after head injury based on Glasgow outcome scale (GOS) as death outcome, vegetative state, severe disability, moderate disability, and good recovery. Patients with moderate disability or recovery were categorized as favorable group and other patients put in unfavorable group. There was no difference in treatment method between both groups. Statistical analysis of all data including GCS on admission, age, sex, results of measurement of interlukines and their relation with patient outcome was conducted by SPSS software (version 18) using the Spearman's rho, Independent T-Test, Mann-Whitney Test. Differences were considered statistically significant at $\mathrm{P} \leq 0.05$.

\section{Results}

From a total of 44 patients included in this study, 97.7\% were male and the rest were female with the mean ages of $33.7 \pm 17.6$ years. Accidents caused by motor vehicle crashes (97.7\%) were the most common cause of trauma. Results of patients outcome at 6 months after head injury based on GOS showed that $56.4 \%$ of the patients died, $2.6 \%$ had vegetative life, $25.6 \%$ severe disability, $10.3 \%$ moderate disability, and 5.1\% good recovery. Based on the previous classification, it can be said that $15.4 \%$ had favorable and $84.6 \%$ unfavorable outcome. Mean ages in favorable and unfavorable outcome groups were $36.7 \pm 8.6$ and $33.1 \pm$ 17.5 , respectively and there was no statistically significant correlation between mean age of groups. Mean GCS at admission time in group with favorable outcome was $5.7 \pm$ 1.7, while in other group, mean GCS was $5.1 \pm 1.5$ suggesting that there is no statistically significant correlation. Serum levels of interleukin $8(\mathrm{P}<0.01)$ and interleukin 6 $(\mathrm{P}<0.03)$ were significantly higher in groups with unfavorable outcomes comparing to favorable outcome; however, no statistically significant difference was observed in serum level of IL -10 in the two groups (Table 1).

Table 1 displays that the mean of serum level of interleukins 6 and 8 in group with favorable outcome (85.251.6 $\mathrm{pg} / \mathrm{mL}$ and 52.231.9 $\mathrm{pg} / \mathrm{mL}$, respectively) that was less than that of group with unfavorable outcome (162.3141.1 pg/mL and $173.6257 .3 \mathrm{pg} / \mathrm{mL}$ ).Studying the correlations between serum level of interleukins at time of measurement and GCS on admission, the results of Spearman correlation coefficient test revealed a statistically negative correlation between GCS at admission and serum level of interleukin $8(\mathrm{P}<0.01)$, no statistically correlation was found between GCS at admission and serum level of IL 6 and IL 10 (Table 2).

Table 1. Serum Levels of Interleukins in the Traumatic Brain Injury (TBI) Patients per Outcome ${ }^{\mathrm{a}, \mathrm{b}}$

\begin{tabular}{lccc}
\hline $\begin{array}{l}\text { Interleukin, } \\
\text { pg/mL }\end{array}$ & $\begin{array}{c}\text { Favorable } \\
\text { Outcome }\end{array}$ & $\begin{array}{c}\text { Unfavorable } \\
\text { Outcome }\end{array}$ & $\begin{array}{c}\text { P-Value } \\
\text { c }\end{array}$ \\
\hline IL-6 & $85.2 \pm 51.6$ & $162.3 \pm 141.1$ & 0.03 \\
IL-8 & $162.3 \pm 141.1$ & $173.6 \pm 257.3$ & 0.01 \\
IL_10 & $52.2 \pm 31.9$ & $83.9 \pm 79.4$ & 0.9 \\
\hline
\end{tabular}

\footnotetext{
a Abbreviation: IL, Interleukin.

$\mathrm{b}$ Values are expressed as Mean \pm SE.

${ }^{\mathrm{C}} \mathrm{P}$ value obtained by Independent $\mathrm{T}$-test for the difference between the two groups.
} 
Table 2. Relationship Between GCS at Admission With Serum Levels of Interleukins

\begin{tabular}{lcc}
\hline Variables & R & P-Value \\
\hline GCS IL-6 & -0.33 & 0.05 \\
GCS IL-8 & -0.43 & 0.01 \\
GCS IL-10 & 0.08 & 0.6 \\
\hline
\end{tabular}

\section{Discussion}

Relationship between clinical status and neuro inflammation was found through measuring inflammatory markers in plasma and cerebrospinal fluid (CSF) (17). Due to importance of inflammation process in clinical status of patient in this study, we attempted to find more information about the role of cytokines including interleukins 6, 8 and 10 in clinical outcome after head injury by measuring serum levels in patients with severe TBI. Our study showed that the mean serum level of IL- 6 in patients with unfavorable outcome was more than that of patients with favorable outcome. Results of a study by Chiaretti et al. were compatible with those of our study. They reached this conclusion by evaluating the levels of this interleukin in CSF and serum of children with severe TBI that increased in IL-6 expression which was indicative of poor clinical outcome (18). Moreover, Antunes et al. observed a remarkable difference in mean plasma levels of IL- 6 in both favorable and unfavorable outcomes; the highest levels of IL-6 was observed in patients with worst clinical course (19). In a study by Kalabalikis et al. on levels of IL-6 in serum of children with head injury, no relationship was found between IL-6 and patient outcome (20). However, the results of several studies on measuring IL-6 levels in CSF of TBI patients showed that increased levels of this interleukin was associated with favorable clinical outcome in patients $(16,21)$. In addition, IL-6 levels in CSF might be associated with favorable clinical outcome whereas measuring serum levels indicated association with unfavorable clinical outcome. Such results show the complexity of neuroinflammation response to TBI and emphasize the fact that cytokines can play multiple roles under different conditions in CSF and peripheral blood circulation (8). In present study, measuring serum levels of interleukin-8 and its relationship with patient outcome revealed that the mean serum level of IL-8 in patients with unfavorable outcome was more than that of patients with favorable outcome. A research by Michael Whalen and colleagues also showed remarkable increase in levels of IL-8 in CSF of children with severe head injury. These high concentrations were associated with mortality in children (15). According to our results, although the mean serum level of IL-10 in patients with unfavorable outcome was more than that of patients with favorable outcome, this difference was not statistically significant. In this regard, the results of a study by Shiozaki et al. showed that IL-10 concentration in CSF in patients with unfavorable outcome was remarkably higher than that of patients with favorable outcome (22). Considering the relationship between these interleukins and patient outcome, Bernd Maier et al. found no significant relationship between interleukins 6,8 and 10 and clinical outcome (based on GOS) 6 months after head injury (23). Similar results were observed in a study by Hayakata and colleagues (24). Overall, it is concluded that the inflammatory proteins according to when and where they are released with respect to their concentration can have both beneficial and detrimental effects (25). The results of present study on relationship between serum levels of interleukins and severe head injury based on GCS indicated that only serum level of IL-8 was negatively correlated with GCS on admission. However, Hayakata et al. found no significant correlation between peaks of IL-8 concentration in CSF and GCS on admission in TBI patients in their study (24). Neuroinflammation occurs in response to traumatic brain injury. This response can affect patient clinical outcome by inflammatory markers such as interleukins. Based on findings of our study, increased levels of interleukins 6 and 8 in first hours after severe TBI were associated with unfavorable clinical outcome. Therefore, measuring serum levels of these interleukins may be considered as a marker for predicting patient clinical outcome following head injury. We hope that measuring and regulating neuro inflammatory response by these markers could be helpful in improving therapeutic strategies for TBI patients to reduce the rate of mortality and morbidity.

\section{Acknowledgements}

We thank the Clinical Research Development Unit in Poursina Hospital for their cooperation and Ms. Fatemeh Javadi for editing the manuscript.

\section{References}

1. Myburgh JA, Cooper DJ, Finfer SR, Venkatesh B, Jones D, Higgins A, et al. Epidemiology and 12-month outcomes from traumatic brain injury in australia and new zealand. J Trauma. 2008;64(4):854-62.

2. Langlois JA, Rutland Brown W, Thomas KE. Traumatic brain injury in the United States: emergency department visits, hospitalizations, and deaths.USA: Department of Health and Human Services, Centers for Disease Control and Prevention, National Center for Injury Prevention and Control; 2006.

3. McIntosh TK, Smith DH, Meaney DF, Kotapka MJ, Gennarelli TA, Graham DI. Neuropathological sequelae of traumatic brain injury: relationship to neurochemical and biomechanical mechanisms. Lab Invest. 1996;74(2):315-42.

4. Gentile NT, McIntosh TK. Antagonists of excitatory amino acids and endogenous opioid peptides in the treatment of experimental central nervous system injury. Ann Emerg Med. 1993;22(6):1028-34.

5. Faden AI, Demediuk P, Panter SS, Vink R. The role of excitatory amino acids and NMDA receptors in traumatic brain injury. Science. 1989;244(4906):798-800.

6. Hall ED, Detloff MR, Johnson K, Kupina NC. Peroxynitrite-mediated protein nitration and lipid peroxidation in a mouse model of traumatic brain injury. J Neurotrauma. 2004;21(1):9-20.

7. Morganti-Kossmann MC, Satgunaseelan L, Bye N, Kossmann T. 
Modulation of immune response by head injury. Injury. 2007; 38(12):1392-400.

8. Oda S, Hirasawa H, Shiga H, Nakanishi K, Matsuda K, Nakamua M. Sequential measurement of IL-6 blood levels in patients with systemic inflammatory response syndrome (SIRS)/sepsis. Cytokine. 2005;29(4):169-75.

9. Roumen RM, Hendriks T, van der Ven-Jongekrijg J, Nieuwenhuijzen GA, Sauerwein RW, van der Meer JW, et al. Cytokine patterns in patients after major vascular surgery, hemorrhagic shock, and severe blunt trauma. Relation with subsequent adult respiratory distress syndrome and multiple organ failure. Ann Surg. 1993;218(6):769-76.

10. Ziebell JM, Morganti-Kossmann MC. Involvement of pro- and anti-inflammatory cytokines and chemokines in the pathophysiology of traumatic brain injury. Neurotherapeutics. 2010;7(1):22-30.

11. Kumar A, Loane DJ. Neuroinflammation after traumatic brain injury: opportunities for therapeutic intervention. Brain Behav Immun. 2012;26(8):1191-201.

12. Morganti-Kossmann MC, Rancan M, Otto VI, Stahel PF, Kossmann T. Role of cerebral inflammation after traumatic brain injury: a revisited concept. Shock. 2001;16(3):165-77.

13. Winter CD, Pringle AK, Clough GF, Church MK. Raised parenchymal interleukin- 6 levels correlate with improved outcome after traumatic brain injury. Brain. 2004;127(Pt 2):315-20.

14. Goodman JC, Van M, Gopinath SP, Robertson CS. Pro-inflammatory and pro-apoptotic elements of the neuroinflammatory response are activated in traumatic brain injury. Acta Neurochir Suppl. 2008;102:437-9.

15. Whalen MJ, Carlos TM, Kochanek PM, Wisniewski SR, Bell MJ, Clark RS, et al. Interleukin-8 is increased in cerebrospinal fluid of children with severe head injury. Crit Care Med. 2000; 28(4):929-34.

16. Singhal A, Baker AJ, Hare GM, Reinders FX, Schlichter LC, Moulton RJ. Association between cerebrospinal fluid interleukin-6 concentrations and outcome after severe human traumatic brain injury. J Neurotrauma. 2002;19(8):929-37.

17. Hopkins SJ, McMahon CJ, Singh N, Galea J, Hoadley M, Scarth S, et al. Cerebrospinal fluid and plasma cytokines after subarachnoid haemorrhage: CSF interleukin-6 may be an early marker of infection. J Neuroinflammation. 2012;9:255.

18. Chiaretti A, Genovese O, Aloe L, Antonelli A, Piastra M, Polidori $\mathrm{G}$, et al. Interleukin 1beta and interleukin 6 relationship with paediatric head trauma severity and outcome. Childs Nerv Syst. 2005;21(3):185-93.

19. Antunes AA, Sotomaior VS, Sakamoto KS, de Camargo Neto CP Martins C, Aguiar LR. Interleukin-6 plasmatic levels in patients with head trauma and intracerebral hemorrhage. Asian J Neurosurg. 2010;5(1):68-77.

20. Kalabalikis P, Papazoglou K, Gouriotis D, Papadopoulos N, Kardara $\mathrm{M}$, Papageorgiou F, et al. Correlation between serum IL- 6 and CRP levels and severity of head injury in children. Intensive Care Med. 1999;25(3):288-92.

21. Chiaretti A, Antonelli A, Mastrangelo A, Pezzotti P, Tortorolo L, Tosi F, et al. Interleukin- 6 and nerve growth factor upregulation correlates with improved outcome in children with severe traumatic brain injury.J Neurotrauma. 2008;25(3):225-34.

22. Shiozaki T, Hayakata T, Tasaki O, Hosotubo H, Fuijita K, Mouri T, et al. Cerebrospinal fluid concentrations of anti-inflammatory mediators in early-phase severe traumatic brain injury. Shock. 2005;23(5):406-10.

23. Maier B, Schwerdtfeger K, Mautes A, Holanda M, Muller M, Steudel WI, et al. Differential release of interleukines 6,8 , and 10 in cerebrospinal fluid and plasma after traumatic brain injury. Shock. 2001;15(6):421-6.

24. Hayakata T, Shiozaki T, Tasaki O, Ikegawa H, Inoue Y, Toshiyuki F, et al. Changes in CSF S100B and cytokine concentrations in earlyphase severe traumatic brain injury. Shock. 2004;22(2):102-7.

25. Mukherjee S, Katki K, Arisi GM, Foresti ML, Shapiro LA. Early TBIInduced Cytokine Alterations are Similarly Detected by Two Distinct Methods of Multiplex Assay. Front Mol Neurosci. 2011;4:21. 